\title{
Detection of QRS Complexes Using Wavelet Transforms and Golden Section Search
}

\author{
Wenli Chen Zhiwen Mo Wen Guo \\ College of Mathematic and Software Science, Sichuan Normal University, Chengdu 610068, China
}

\begin{abstract}
The extraction and identification of ECG (electrocardiogram) signal characteristic parameters are the basis of ECG analysis and diagnosis. The fast and precise detection of QRS complexes is very important in ECG signal analysis, for it is the precondition of the correlative parameters calculation and diagnosis. In this paper, the modulus maxima of wavelet transform was applied to the QRS complexes detection of ECG signal firstly. Once misdetections or fail-detections happen, we used the Golden Section Search to adjust the thresholds of maxima determination. The correct detection rate of the QRS complexes is up to $99.6 \%$ based on MIT-BIH ECG data.
\end{abstract}

Keywords: ECG Signal QRS Complexes Wavelet Transformation Golden Section Search

\section{Introduction}

QRS complexes, $\mathrm{P}$ and $\mathrm{T}$ waves are important key features of electrocardiogram (ECG), so automatic detection of ECG waves (QRS complexes, P and T wave) is important to cardiac disease analysis and diagnosis. The detection of ECG signal is began form QRS complexes. Calculation and analysis of heart rate and $\mathrm{P}$ and $\mathrm{T}$ waves are based on the precise detection of QRS complexes, so that it is essential for the ECG automatic detection.

Many methods mostly non-syntactic have been used for QRS detection [1-5]. These methods mainly suffer from three problems: (1) QRS waveform varies from patient to patient and depends on the state of the patient; (2) noise and QRS complexes pass bands overlap; (3) without post-filtering, we can introduce significant modifications in the shape of the signal, generating false detection result. So the effect of a single threshold which is used to detect the QRS complexes is limited for the variability of QRS waveforms for different beats of the same subject [6].

Wavelet transform is a good time-frequency characteristics of local signal analysis. It is not only multi-channel filtering characteristics, but also has a good capacity of shown the local signal characterization. So it is suitable for the detection of the signal feature points or transient information. Golden section search [7] calculates simply. It can be used to adjust the detection thresholds which play a major role in detection different ECG signals, thereby improving the accuracy rate. This paper used the wavelet transform combined with the golden section search in the QRS complexes real-time detection and made a good experimental result.

\section{Theory}

\subsection{Wavelet transform}

The wavelet transform of a signal $f(x)$ is defined as

$W_{s} f(x)=f(x) * \psi(x)=\frac{1}{s} \int_{-\infty}^{+\infty} f(t) \varphi\left(\frac{x-t}{s}\right) d t$

where $\mathrm{S}$ is scale factor. $\quad \psi_{\mathrm{s}}(x)=\frac{1}{\mathrm{~S}} \psi\left(\frac{x}{\mathrm{~s}}\right) \quad$ is the dilation of a basic wavelet $\psi(x)$ by the scale factor $S$. Let $s=2^{j}(j \in Z)$, then the wavelet transform is called dyadic wavelet transform [8]. The dyadic wavelet transform of a digital signal $f(n)$ can be calculated with Mallat algorithm [9] as follows:

$$
\begin{aligned}
& \mathrm{S}_{2^{\mathrm{j}}} f(n)=\sum_{k \in Z} h_{k} S_{2^{j-1}} f\left(n-2^{j-1} k\right) \\
& \mathrm{W}_{2^{\mathrm{j}}} f(n)=\sum_{k \in Z} g_{k} W_{2^{j-1}} f\left(n-2^{j-1} k\right)
\end{aligned}
$$

where $S_{2^{j}}$ is a smoothing operator and $s_{2^{0}} f(n)=d_{n}, d_{n}$ is the digital signal to be analyzed [10], which is the ECG signal used in this paper. $W_{2^{j}} f(n)$ is the wavelet transform of digital signal $f(n) .\left\{h_{k} \mid k \in Z\right\}$ and $\left\{g_{k} \mid k \in Z\right\}$ are coefficients of a low-pass filter $H(w)$ and high-pass filter $G(w)$, respectively; that means

$$
H(w)=\sum_{k \in Z} h_{k} e^{-i k w} \quad G(w)=\sum_{k \in Z} g_{k} e^{-i k w}
$$


We used the Mallat algorithm of the quadratic spline wavelet to decompose the ECG signal whose sampling rate is 360 points/second.

\subsection{Golden section search}

The golden section search is a technique for finding the extremum (minimum or maximum) of a unimodal function by successively narrowing the range of values inside which the extremum is known to exist. The technique derives its name from the fact that the algorithm maintains the function values for triples of points whose distances form a golden ratio.

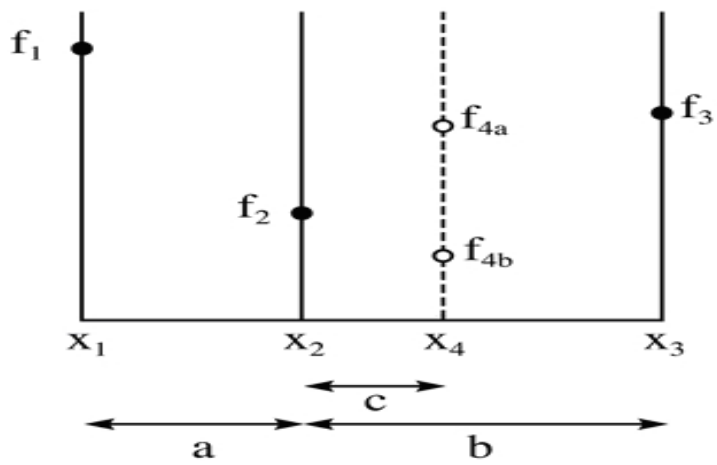

Fig. 1: Diagram of a golden section search

The diagram illustrates a single step in the technique for finding a minimum. The functional values of $f(x)$ are on the vertical axis, and the horizontal axis is the $x$ parameter. The value of $f(x)$ has already been evaluated at the three points: $x_{1}, x_{2}$, and $x_{3}$. Since $f_{2}$ is smaller than either $f_{1}$ or $f_{3}$, it is clear that a minimum lies inside the interval from $x_{1}$ to $x_{3}$.

The next step in the minimization process is to "probe" the function by evaluating it at a new value of $x$, namely $x_{4}$. It is most efficient to choose $x_{4}$ somewhere inside the largest interval, i.e. between $x_{2}$ and $x_{3}$. From the diagram, it is clear that if the function yields $f_{4 a}$ then a minimum lies between $x_{1}$ and $x_{4}$ and the new triplet of points will be $x_{1}, x_{2}$, and $x_{4}$. However if the function yields the value $f_{4 b}$ then a minimum lies between $x_{2}$ and $x_{3}$, and the new triplet of points will be $x_{2}, x_{4}$, and $x_{3}$. Thus, in either case, we can construct a new narrower search interval that is guaranteed to contain the function's minimum.

When applied the golden section search, the continuity and differentiability of the function are not required. And it isn't necessary to write the analytical expression of the function. We only need to find the corresponding function values of the chosen insertion points. The golden section search has a broad scope to be applied in.

\section{Extraction of QRS complexes's feature based on wavelet transform and golden section search}

\subsection{Choice of wavelet mother function}

Because there are many wavelet mother functions to be chosen for wavelet transform, choice of fit wavelet mother function is very important for ECG signal detection. First, as wavelet transform plays a role of band-pass filters in the signal processing. Symmetry and anti-symmetry are equivalent to the linear phase and generalized linear phase, respectively. If a bandpass filter isn't linear phase or generalized linear phase, it'll distort the signal. To avoid distortion of signal, the wavelet function and its dual wavelet function being symmetrical and anti-symmetrical should be chosen. And consideration of the theory and practical application aspects, the mother wavelet functions of compactly supported subsets are the best choices. However, the orthogonal wavelet functions of real value and compactly supported subsets are not symmetrical or anti-symmetrical except spline wavelet transforms [11] (Harr wavelet is linear spline wavelet). Second, consideration of accuracy and speed, Harr wavelet function uses a group of beelines to approximate the signal, and the quadratic or cubic spline wavelet uses a group of quadratic or cubic curve to approximate the signal. So the quadratic spline wavelet is a good choice for ECG signal detection.

\subsection{Removal of the baseline drift}

As a physiological cardiac electrical activity in the performance of the human body surface, ECG signal is weak, whose range is between $110 \mu \mathrm{v}-4 \mathrm{mv}$.So it is easily affected by environment. ECG signal mainly contains noises of seven different types [12], namely frequency interference, baseline drift, electrode contact noise, polarization noise, muscle noise, the internal amplifier noise and motor artifacts. $50 \mathrm{~Hz}$ or near the power line interference and the baseline drift which is below $1 \mathrm{~Hz}$ are the main sources of the noises.

We used the median filter to eliminate baseline drift of ECG signal [13]. The process is as follows:

1) Let the ECG signal $S$ go across the 200-ms median filter. We received the signal $S_{1}$ which was eliminated the QRS complexes and $\mathrm{P}$ wave.

2) Let the signal $S_{1}$ go across the 600 -ms median filter. We received the signal $S_{2}$ which $T$ wave was eliminated. So $S_{2}$ is the baseline drift signal which didn't have QRS complexes, $\mathrm{P}$ and $\mathrm{T}$ waves. 
3) After the displacement and subtraction $\mathrm{S}_{2}$ from original signal $S$, we received the signal $S^{*}$ which was eliminated the baseline drift. The results are shown by figure 2 :

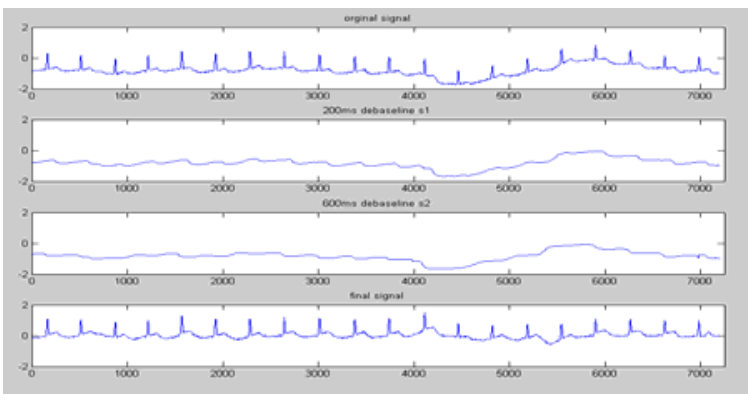

Figure 2: Elimination of baseline drift.

\subsection{Detection of $\mathbf{R}$ peak based on wavelet transform and golden section search}

The signal S' which was eliminated the baseline drift was decomposed by wavelet transform to detail signals of different scales, namely detail signals $d 1, d 2$, $d 3, d 4$ of different scales $2^{1}, 2^{2}, 2^{3}, 2^{4}$, respectively. The result is shown by figure 3 . The high frequency noises like frequency interference and muscle noise are mainly in the $d 1$ and $d 2$. The frequency of QRS complexes is between $10 \mathrm{~Hz}$ and $25 \mathrm{~Hz}$.The energy of QRS complexes is mainly in the $d 3$ and $d 4$. So we only selected scales from $2^{3}$ to $2^{4}$ for the detection of $\mathrm{R}$ peak.

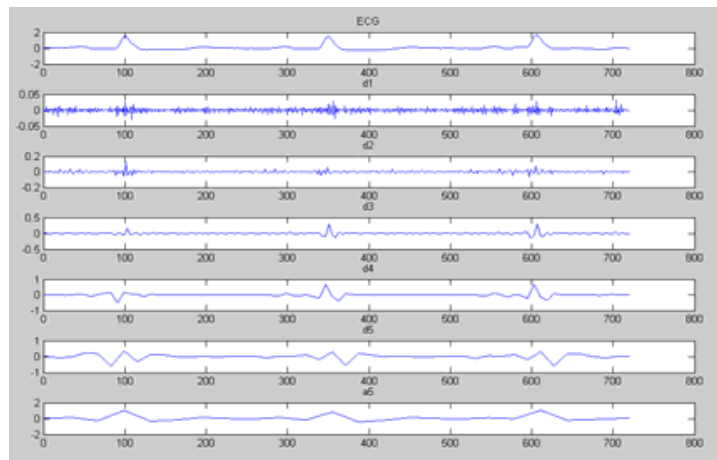

Figure 3: Decomposition of ECG signal at different scales

A method to detection $\mathrm{R}$ peak is as follows:

Step 1: We detected 7200 sampling points (20 seconds) each time, and used the wavelet transform to decompose the signal to different scales from $2^{1}$ to $2^{4}$.

Step 2: Select the $\beta_{4}$ times of the average modulus maxima at scale $2^{4}$ as the threshold $\varepsilon_{4}$. Find all of the modulus maxima larger than $\varepsilon_{4}$ at scale $2^{4}$ to obtain the location set of the modulus maxima $\left\{n_{k}^{4} \mid k=1, \ldots, N\right\}$.
Step 3: Select the $\beta_{3}$ times of the average modulus maxima at scale $2^{3}$ as the threshold $\varepsilon_{3}$. Find all of the modulus maxima larger than $\varepsilon_{3}$ on the neighborhood of $n_{\mathrm{k}}^{3}$ at scale $2^{3}$ and define its location as $n_{k}^{3}$. If several modulus maxima exist, then the largest one is selected. However, the modulus maxima with its location nearest $n_{\mathrm{k}}^{4}$ will be selected if the largest one isn't larger than 1.2 times of the others. If no modulus maxima exists, then set $n_{\mathrm{k}}^{3}$ and $n_{\mathrm{k}}^{4}$ to zero. So the location set $\left\{n_{k}^{3} \mid k=1, \ldots, N\right\}$ can be found.

Step 4: $\mathrm{R}$ wave corresponds to a positive maxima-negative minimum pair at scale $2^{3}$. If the interval between $n_{1}^{3}$ and $n_{k}^{3}(k \neq 1)$ is larger than an interval threshold, the maximum (minimum) at $n_{1}^{3}$ is considered the redundant maximum (minimum). If the corresponding modulus maximum (minimum) is redundant, it should be eliminated from the set $n_{k}^{3}$. The selected interval threshold should be approximately the same as the interval of the two modulus maxima created by the widest possible QRS complex in order that wide QRS complex isn't lost and artifacts and noises are mostly eliminated. Generally, the cycle of one QRS complex is between $40 \mathrm{~ms}$ and $120 \mathrm{~ms}$. So the interval threshold is defined as $120 \mathrm{~ms}$, namely 43 sampling points.

Step 5: Find the two nearest positive maximum modulus point and negative minimum modulus point comprise the modulus maximum pair in 43 sampling points. The position of the QRS complex is the midpoint of the modulus maximum pair. So the location set of QRS complexes $\left\{N_{q r s}^{k} \mid k=1,2, \ldots, M, M \leq N\right\}$ can be found.

Step 6: $\mathrm{R}$ peak is peak or though of the largest amplitude in the QRS complex. The peak or though of the largest amplitude in the neighborhood of $N_{\text {qrs }}^{k}(k=1, . ., M)$ is defined as R peak recorded as $N_{r}^{q r s}(k=1, \ldots, M)$. Select the $\alpha$ times of the average maximum amplitude as threshold $\varepsilon$. If the absolute value of $N_{r}^{k}(k=1, \ldots, M)$ is less than $\varepsilon, N_{r}^{k}$ is the mis-detected $\mathrm{R}$ peak which should be eliminated.

Step 7: Calculate the average value $P$ of $\mathrm{R}-\mathrm{R}$ interval. If the distant between the two nearest $\mathrm{R}$ peaks is less than 0.6 times of $P$, we thought falsedetection happened. If the distant between the two nearest $\mathrm{R}$ peaks is larger than 1.5 times of $P$, we thought mis-detection happened. If false-detection and mis-detection didn't happen, the detection was over. Else, go to step 8 . The result is shown by figure 4 .

Step 8: Adjust the value of parameters $\beta_{3}$ and $\beta_{4}$ by golden section search, and go to step 2 . The result of $\mathrm{R}$ peak detection is shown by figure 5 . 


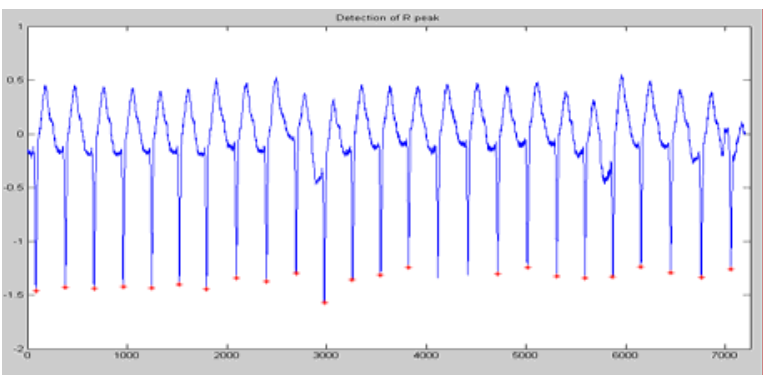

Figure 4: Mis-detection of R peak

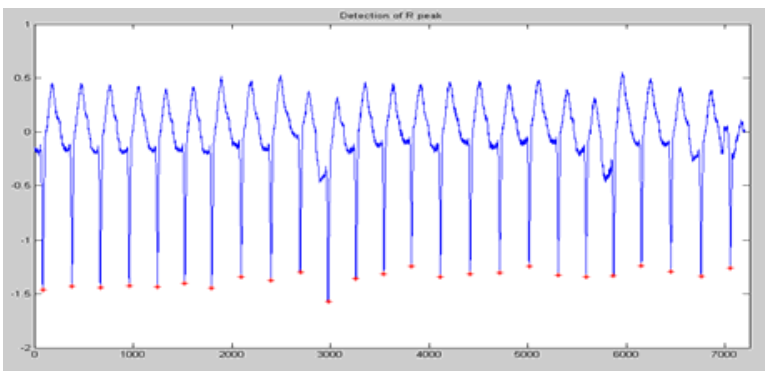

Figure 5: Exact detection of R peak

\section{Discussion}

(1) The onset and offset of the QRS complex can also be detected after the detection of $\mathrm{R}$ peak. The beginning of the $\mathrm{Q}$ wave defines as the onset of the QRS complex, and the ending of the $S$ wave defines as the offset of the QRS complex. Q and S waves are high frequency and low amplitude waves and their energies are mainly at small scale. So we detected Q and $\mathrm{S}$ waves at scale $2^{1}$, and the method is similar to the previous.

(2) Happing of the mis-detection or falsedetection was caused by many factors. If the mistakes were not caused by the values of the parameters $\beta_{3}$ and $\beta_{4}$, the results didn't change no matter how adjust the values. We can use the iteration time to control the processes in order to determine if there are other reasons to mis-detection.

\section{Conclusions}

In order to evaluate the method, we used some records from MIT-BIH arrhythmia database to detect the QRS complexes. The results are shown in table 1 . We obtained good experimental results to most of the ECG data. The method is better than that only used modulus maximum pairs to detect the QRS complexes [14]. Since record 207 has ventricular fibrillation for a long time, and the way of ventricular fibrillation detection [15] is different from the ordinary method to detect the ECG signal. The detection rates are low, when we used the method to detect 207.

\begin{tabular}{llllll}
\hline Record & $\begin{array}{l}\text { Total } \\
\text { (beats) }\end{array}$ & $\begin{array}{l}\text { TP } \\
\text { (beats) }\end{array}$ & $\begin{array}{l}\text { FP } \\
\text { (beats) }\end{array}$ & $\begin{array}{l}\text { FN } \\
\text { (beats) }\end{array}$ & $\begin{array}{l}\text { Accuracy } \\
(\%)\end{array}$ \\
\hline 100 & 2267 & 2267 & 0 & 0 & 100 \\
101 & 1865 & 1865 & 0 & 0 & 100 \\
102 & 2179 & 2177 & 0 & 2 & 99.91 \\
103 & 2078 & 2078 & 0 & 0 & 100 \\
107 & 2124 & 2124 & 5 & 2 & 99.67 \\
109 & 2530 & 2520 & 8 & 2 & 99.60 \\
202 & 2131 & 2124 & 5 & 2 & 99.67 \\
207 & 1856 & 1802 & 35 & 19 & 97.09 \\
230 & 2256 & 2249 & 3 & 4 & 99.68 \\
\hline
\end{tabular}

Table 1: The results of the QRS complexes detection for the MIT-BIH database.

\section{Acknowledgement}

This work is supported by National Nature Science Foundation of China (Grant No. 10671031), and partially Youth Science \& Technology Foundation of Sichuan Province (Grant NO. 07ZQ026-114).

\section{References}

[1] Q. Z. Xie, Y. H. Hu, W. J. Tompkins, NeuralNetwork Based Adaptive Matched Filtering of QRS Detection, IEEE Trans. Biomed. Eng., 39: 317-329, 1992.

[2] F. Gritzali, G. Frangakis, G. Papakonstantinou, Detection of the $\mathrm{P}$ and $\mathrm{T}$ Waves in an ECG, Comput. Biomed. Res., 22: 83-91, 1989.

[3] J. Pan, and W. J. Tompkins, A Real-Time QRS Detection Algorithm, IEEE Trans. Biomed. Eng., 32:230-236, 1985.

[4] P. S. Hamilton and W. J. Tompkins, Quantitative Investigation of QRS Detection Rules Using the MIT/BIH Arrhythmia Database, IEEE Trans. Biomed. Eng., 33: 1157-1165, 1986.

[5] Cuiwei Li, Chongxun Zheng, Changfeng Tai, Detection of ECG Characteristic Points Using Wavelet Transforms, IEEE Trans. Biomed. Eng., 42(1):21-28, 1995.

[6] Zhengrong Yang, A Method of QRS Detection Based on Wavelet Transforms, the Master Thesis of National Sun Yat-Sen University, pp. 614, 2004.

[7] Guangyan Shi, Jiali Dong, Methods of Optimization, Higher Education Press, pp. 44-46, 1999.

[8] S. Mallat and W. L. Hwang, Singularity Detection and Processing with Wavelets, IEEE Trans. Inform. Theory. 38:617-643, 1993.

[9] S. Mallat, Zero-Crossings of a Wavelet Transform, IEEE Trans. Inform. Theory., 37:1019-1033, 1991. 
[10] S. Mallat, Characterization of Signals from Multiscale Edges, IEEE Trans. Pattern Anal. Machine Intell., 14:710-732, 1992.

[11] Wufan Chen, Wavelet Analysis and its Application in the Image Processing, Published by Science Press, pp. 36-58.2002.

[12] Wei Hong, Research in Algorithms of ECG Waveforms Classification, Master Thesis of Zhejiang University, pp. 9-10, 2001.

[13] P. de Chazal, C. Heneghan, E. Sheridan, R. Reilly, P. Nolan, M. O'Malley, Automated Processing of the Single-Lead Electrocardiogram for the Detection of Obstructive Sleep Apnoea, IEEE Trans. Biomed. Eng., 50( 6): 686-689, 2003.

[14] Wei Lin, Xiaoming Wu, Weizong Zhu, Jing Zhou, Using the Modulus Maximum of Wavelet Transform to Detect the ECG Features, Chinese Medical Equipment Journal, 10:13-14, 2004.

[15] Wenyu Ye, Shufeng Li, Xiaoyu Wu, Jilun Ye, Detection of Muti-lead ECG Character Points and Assessment Based on a Reference Database, IEEE EMBS 27 ${ }^{\text {th }}$ Annual Conference, pp. 38953898, 2005. 